\title{
Evaluation of various selective media for the detection of Pseudomonas species in pasteurized milk
}

\author{
J. A. Van Tassell, N. H. Martin, S. C. Murphy, M. Wiedmann, K. J. Boor, and R. A. Ivy ${ }^{1}$ \\ Milk Quality Improvement Program, Department of Food Science, Cornell University, Ithaca, NY 14853
}

\begin{abstract}
Pseudomonas spp. are common gram-negative, postpasteurization contaminants that contribute to spoilage of pasteurized dairy products. This study evaluated 5 common selective media for detecting Pseudomonas spp. in pasteurized milk. The performance of each selective medium for recovering 12 different Pseudomonas isolates (selected to represent a diversity of pasteurized milk isolates) was compared with that of standard plate count agar pour plates. Pseudomonas isolates showed varying abilities to produce colonies on different selective media. For 2 of 12 isolates, a 48-h incubation time was required for colony formation on any of the media tested. Violet red bile agar and coliform Petrifilm (3M, St. Paul, MN) were less effective than standard plate count agar pour plates at recovering Pseudomonas, regardless of incubation time, and MacConkey agar showed poor detection efficiency compared with SPCP after a 48-h incubation $\left(\mathrm{R}^{2}=0.26\right)$. Therefore, the use of violet red bile agar, MacConkey agar, or coliform Petrifilm may not be sufficient for detecting common Pseudomonas spp. in milk. The methods showing the highest detection efficiencies were crystal violet tetrazolium agar $\left(\right.$ CVTA) pour plates $\left(\mathrm{R}^{2}=0.95\right)$ and CVTA plates inoculated by spiral plating $\left(\mathrm{R}^{2}=0.89\right)$ incubated at $32^{\circ} \mathrm{C}$ for $48 \mathrm{~h}$. Overall, plating milk samples on CVTA followed by a 48 -h incubation at $32^{\circ} \mathrm{C}$ was the most effective selective method for recovering a diversity of Pseudomonas spp. from milk.
\end{abstract}

Key words: Pseudomonas, gram-negative, milk quality, post-pasteurization contamination

\section{INTRODUCTION}

Contamination with psychrotolerant gram-negative bacteria remains a leading cause of fluid milk spoilage and food loss in the United States (Eneroth et al., 2000; Carey et al., 2005; Ranieri and Boor, 2009; Martin et al., 2011). The combination of stringent cleaning and

Received September 20, 2011.

Accepted November 6, 2011.

${ }^{1}$ Corresponding author: rai6@cornell.edu sanitation programs and legal pasteurization regimens (e.g., HTST) effectively minimizes gram-negative spoilage bacteria present in the raw milk. Therefore, gram-negative spoilage is generally an indicator of postpasteurization contamination (Ralyea et al., 1998).

Members of the gram-negative genus Pseudomonas are common in the dairy continuum, and several species are frequently isolated from raw milk (Ternstrom et al., 1993; Jayarao and Wang, 1999). Many dairy-associated Pseudomonas spp. produce degradative enzymes (i.e., proteases and lipases) (Wiedmann et al., 2000; Nicodeme et al., 2005; Boran and Ugur, 2010; De Jonghe et al., 2011) that can produce rancid and bitter offflavors in milk. Reducing the presence of gram-negative spoilage organisms, including Pseudomonas spp., in dairy processing environments and finished products is critical to extending the shelf life of pasteurized dairy products, including fluid milk.

Several bacteriological media are available for selectively detecting gram-negative bacteria in dairy products. Crystal violet tetrazolium agar (CVTA; Frank and Yousef, 2004), MacConkey agar (MAC; Difco, 1998), violet red bile agar (VRBA; Frank and Yousef, 2004), and 3M Coliform Petrifilm (CPETRI, 3M, St. Paul, MN) are examples of gram-negative selective media. Crystal violet tetrazolium agar, MAC, and VRBA contain crystal violet to inhibit the growth of grampositive bacteria, and MAC and VRBA also contain bile salts, which may inhibit some gram-negative organisms; therefore, CVTA is the recommended method for the detection of total gram-negative bacteria in milk (Frank and Yousef, 2004). Violet red bile agar and MAC also contain lactose and a $\mathrm{pH}$ indicator and can thus be used to differentiate non-lactose-fermenters from lactose fermenters, which include coliform bacteria. Coliform Petrifilm is designed to detect specifically coliform bacteria and is widely used in the dairy industry.

The use of VRBA, MAC, or CPETRI as the only method of gram-negative detection by dairy processing facilities may result in false-negative results for the presence of certain gram-negative spoilage organisms (e.g., Pseudomonas spp.) that may be sensitive to the additional selective agents (e.g., bile salts) in these media. Although a previous study tested 2 strains of Pseu- 
Table 1. Isolates used in this study and species identification(s) based on 16S rDNA sequencing

\begin{tabular}{llc}
\hline & & $\begin{array}{c}\text { 16S rDNA } \\
\text { sequence } \\
\text { FSL ID }\end{array}$ \\
\hline FSL R5-202 & Species identification $^{2}$ & $1 \%)$ \\
FSL R5-300 & Pseudomonas lurida/poae & 100 \\
FSL R5-318 & Pseudomonas proteolytica/brenneri & 99.2 \\
FSL R5-504 & Pseudomonas vranovensis & 99.9 \\
FSL R5-555 & Pseudomonas tolaasii & 100 \\
FSL R5-695 & Pseudomonas migulae & 99.9 \\
FSL R5-779 & Pseudomonas stutzeri/balearica & 100 \\
FSL W6-286 & Pseudomonas rhizosphaerae & 100 \\
FSL W6-524 & Pseudomonas poae/lurida & 99.2 \\
FSL W6-987 & Pseudomonas brenneri/gessardii/rhodesiae & 100 \\
FSL W7-098 & Pseudomonas fragi & 100 \\
FSL W7-425 & Pseudomonas plecoglossicida & 100 \\
\hline
\end{tabular}

${ }^{1}$ Cornell Food Safety Lab isolate designation. More isolate information can be found at www.microbetracker. net using the full isolate designation (e.g., FSL R5-202).

${ }^{2}$ Based on sequence identity to respective type strain sequence obtained using Seqmatch function in Ribosomal Database Project (http://rdp.cme.msu.edu/).

domonas on 3 types of selective gram-negative media (Glueck-Chaloupka and White, 2004), little information exists on the direct comparison of the abilities of gram-negative selective media to quantitatively recover a diverse set of Pseudomonas dairy isolates. To this end, this study directly compared the performance of several gram-negative selective media, including coliform media, in recovering multiple strains of Pseudomonas from milk. From a collection of over 200 Pseudomonas isolates, collected mostly from raw and conventionally pasteurized milk, 12 were chosen to represent a diversity of $16 \mathrm{~S}$ rDNA sequence types. This study provides the dairy industry a comprehensive analysis of media options for detecting post-pasteurization contamination by Pseudomonas spp.

\section{MATERIALS AND METHODS}

\section{Selection of Pseudomonas Isolates}

The Pseudomonas isolates tested here $(\mathrm{n}=12$; Table 1) were chosen from over 200 Pseudomonas milk isolates (excluding isolates identified as closely related to potentially pathogenic Pseudomonas aeruginosa) cataloged in the PathogenTracker database (available at http://www.microbetracker.net). To ensure sequence diversity, partial $16 \mathrm{~S}$ rDNA sequencing was performed as described previously (Durak et al., 2006; Huck et al., 2007) on the Pseudomonas isolates. Partial 16S rDNA sequences from the 12 Pseudomonas isolates and from representative Pseudomonas type strains obtained from the Ribosomal Database Project (RDP; http:// rdp.cme.mdsu.edu/) were aligned and trimmed with MegAlign (version 8.0.2, DNAStar, Madison WI), and a phylogenetic tree was produced using the maximum parsimony method in PAUP*4.0 with 500 bootstrap replications. A Citrobacter freundii partial 16S sequence was used as an outgroup in the phylogenetic analysis. Based on the phylogenic analysis, most of the 12 Pseudomonas isolates grouped with different type strains into unique phylogenetic clades (Figure 1), indicating that the isolates chosen represent a diverse selection within the genus Pseudomonas.

\section{Comparison of Selective Media}

Frozen stocks of the bacterial isolates were obtained from the Cornell University Food Safety Laboratory (CU-FSL) isolate collection. Cultures were streaked from $-80^{\circ} \mathrm{C}$ frozen stock onto brain heart infusion (Difco, Sparks, MD) agar and incubated for $18 \mathrm{~h}$ at $32^{\circ} \mathrm{C}$. A colony from each strain was used to inoculate $5 \mathrm{~mL}$ of sterile brain heart infusion broth in 16-mm glass test tubes, which were then incubated for $48 \mathrm{~h}$ with aeration (shaking at $220 \mathrm{rpm}$ ) at $32^{\circ} \mathrm{C}$. A $1-\mathrm{mL}$ aliquot of the culture was then centrifuged at $14,000 \times$ $g$ and the supernatant was removed. The resulting cell pellet was resuspended in $1 \mathrm{~mL}$ of phosphate dilution buffer (Weber Scientific, Trenton, NJ), diluted to 1,000 to $10,000 \mathrm{cfu} / \mathrm{mL}$, and inoculated into commercially purchased UHT pasteurized milk (1\% milk; Organic Valley, La Farge, WI) for an approximate inoculum of 2 to $3 \log \mathrm{cfu} / \mathrm{mL}$. Inocula were enumerated by spiral plating the appropriate dilution on SPC agar (EMD, Gibbstown, NJ), followed by incubation at $32^{\circ} \mathrm{C}$ for 24 to $48 \mathrm{~h}$. Inoculated UHT milk was incubated at $6^{\circ} \mathrm{C}$ for $48 \mathrm{~h}$ to simulate refrigerated storage conditions. After a 48 -h incubation at $6^{\circ} \mathrm{C}$, appropriate dilutions of inoculated UHT samples prepared in phosphate dilution buffer were plated in duplicate on (1) SPC 


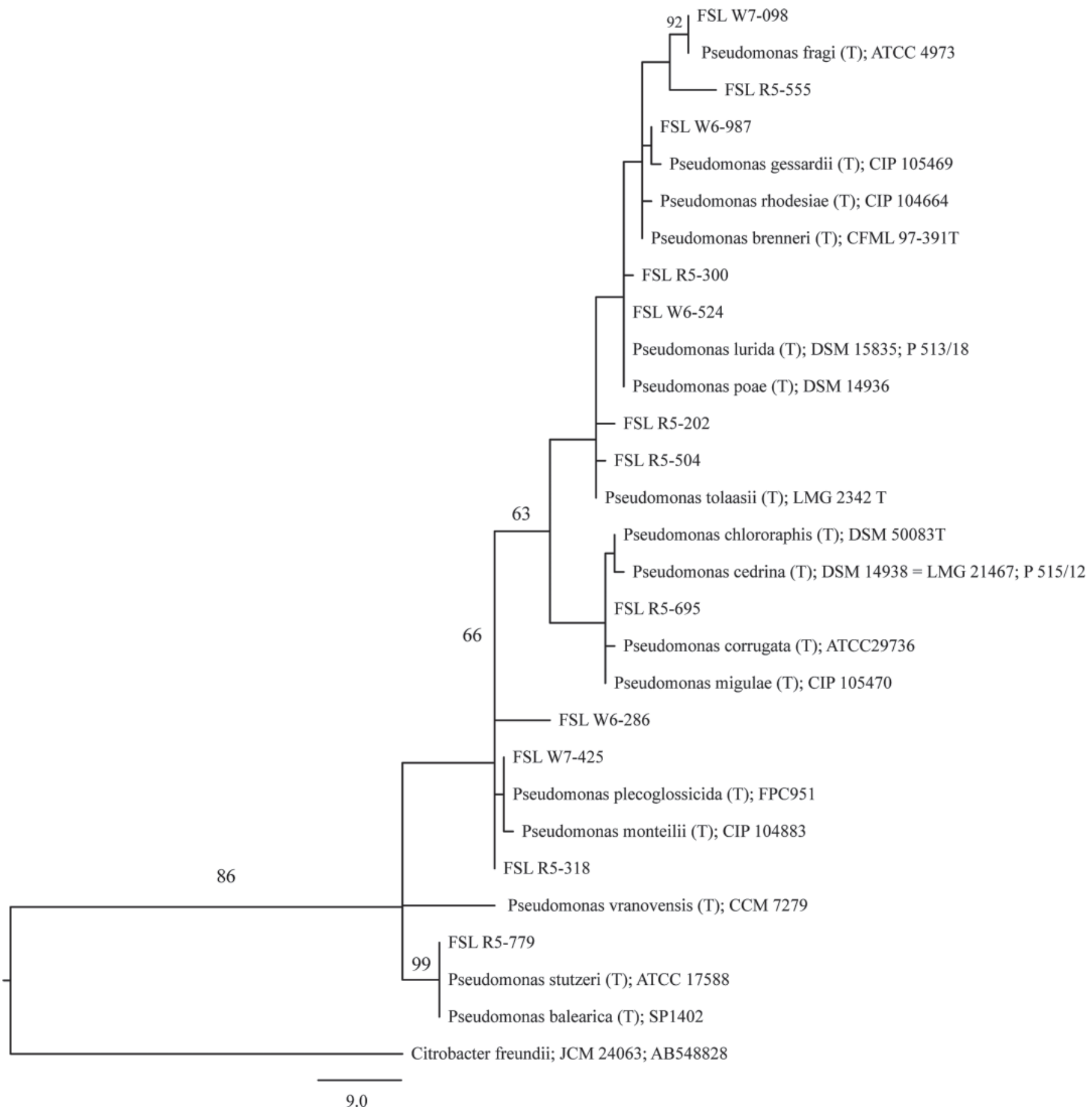

Figure 1. Maximum parsimony phylogenic tree of partial 16S rDNA sequences from a selection of dairy-associated Pseudomonas isolates, indicated by strain designations that start with "FSL" and relevant Pseudomonas spp. type strains obtained from the Ribosomal Database Project (http://rdp.cme.msu.edu/). The Citrobacter freundii type strain sequence was included as an outgroup. Numerical node labels represent the percentage of bootstrap replicates $(n=500)$ that supported the respective node. Only bootstrap values $>50$ are shown. Scale represents estimated substitutions per site.

pour plates (SPCP); (2) SPC plates inoculated by spiral plating (SPCS); (3) CVTA [plate count agar (EMD, Gibbstown, NJ) with 2,3,5-triphenyltetrazolium chloride (Sigma-Aldrich, St. Louis, MO)] pour plates
(CVTAP); (4) CVTA plates inoculated by spiral plating (CVTAS); (5) VRBA plates inoculated by pour plating (Difco, Sparks, MD); (6) VRBA with an overlay of approximately $5 \mathrm{~mL}$ of VRBA agar (VRBAO); (7) 
CPETRI (3M); or (8) MAC (Difco) inoculated by spiral plating. All media were incubated at $32^{\circ} \mathrm{C}$ for $48 \mathrm{~h}$, with enumeration after 24 and $48 \mathrm{~h}$. Three biological replications of the experiment were completed.

\section{Statistical Analyses}

Separate one-way ANOVA and post hoc Tukey's honestly significant difference tests were performed to test for (1) differences in final counts on different media among different Pseudomonas isolates and (2) differences among isolates for survival and growth in UHT milk at $6^{\circ} \mathrm{C}$ over $48 \mathrm{~h}$. Linear regression was used to fit counts on each medium to counts on the nonselective control agar, SPCP. The $\mathrm{R}^{2}$ value of the linear regression, a measure of how well each medium could predict SPCP, was used as a measure of detection efficiency at 24 or 48 h. For example, a higher $\mathrm{R}^{2}$ value (maximum is 1.0) indicates higher predictability for SPCP results and, accordingly, a greater efficiency in recovering Pseudomonas from milk. All statistical analyses were performed using JMP (version 9.0.0, SAS Institute Inc., Cary, NC).

\section{RESULTS AND DISCUSSION}

Post-pasteurization contamination with Pseudomonas spp. represents an important contributor to the spoilage of dairy products. Therefore, testing final product for the presence of Pseudomonas spp. is critical to ensuring the quality and increasing the shelf life of pasteurized dairy products. We examined the efficiency of 5 different selective media and 2 incubation times for the detection of Pseudomonas spp. from milk. Our results indicated that (1) commonly used gram-negative selective media showed differences in detection of Pseudomonas spp. in milk, (2) incubation of selective agar for $48 \mathrm{~h}$ after plating is required for reliable detection of Pseudomonas spp. from milk, and (3) different Pseudomonas isolates differed in their abilities to grow on selective media.

\section{Commonly Used Gram-Negative Selective Media Showed Differences in Detection of Pseudomonas spp. in Milk}

Isolates were inoculated into UHT milk, incubated for $48 \mathrm{~h}$ at $6^{\circ} \mathrm{C}$, and then enumerated on SPC pour plates (SPCP) and spiral plates (SPCS) as well as on 5 different selective media. Colony counts on the different media were determined after incubation for 24 and 48 $\mathrm{h}$ (all counts are presented in Supplementary Table S1, available at http://www.journalofdairyscience.org/). After $24 \mathrm{~h}$, a significant difference was observed among media tested for enumerating Pseudomonas spp. $(P<$ 0.0001; ANOVA). Specifically, the nonselective media SPCP and SPCS showed the highest mean counts (both showed $3.74 \log \mathrm{cfu} / \mathrm{mL}$ ), followed by CVTAP, MAC, and CVTAS $(3.25,3.21$, and $3.08 \log \mathrm{cfu} / \mathrm{mL}$, respectively). Violet red bile agar had the lowest mean counts after $24 \mathrm{~h}(1.59$ and $1.38 \log \mathrm{cfu} / \mathrm{mL}$ for VRBA and VRBAO, respectively).

To determine the efficiency of each selective agar for enumerating Pseudomonas spp. grown in milk at $6^{\circ} \mathrm{C}$, linear regression was used to compare bacterial counts on each medium with SPCP counts. An $\mathrm{R}^{2}$ value close to 1.0 indicates maximum detection efficiency. After $24 \mathrm{~h}$, among the selective media tested, MAC counts most accurately predicted SPCP counts at $24 \mathrm{~h}\left(\mathrm{R}^{2}=\right.$ $0.66)$, followed by CVTAP $\left(\mathrm{R}^{2}=0.56\right)$ and CVTAS $\left(\mathrm{R}^{2}\right.$ $=0.36)$ (Figure 2a). Violet red bile agar showed the lowest detection efficiency; VRBA had an $\mathrm{R}^{2}=0.13$, and VRBAO had an $\mathrm{R}^{2}=0.29$ (Figure 2a).

After $48 \mathrm{~h}$, the general gram-negative selective media CVTAP and CVTAS showed the highest efficiencies $\left(\mathrm{R}^{2}=0.95\right.$ and 0.89 , respectively; Figure $\left.2 \mathrm{~b}\right)$ in recovering Pseudomonas. Furthermore, mean counts on these media were not significantly different from those on SPCS or SPCP $(P>0.05$; Tukey honestly significant difference test), whereas coliform media (i.e., CPETRI, VRBA, and VRBO) had mean counts that were significantly lower than those on SPCS and SPCP. Thus, CVTA clearly performed best at detecting a diversity of Pseudomonas spp. from milk. Surprisingly, MAC showed a lower $R^{2}$ value after $48 \mathrm{~h}\left(\mathrm{R}^{2}=0.26\right)$ than after $24 \mathrm{~h}\left(\mathrm{R}^{2}=0.66\right)$, although the $\mathrm{R}^{2}$ of MAC after $48 \mathrm{~h}$ was higher than that of VRBA, VRBAO, and CPETRI, which had $\mathrm{R}^{2}$ values of $0.02,0.06$, and 0.13 , respectively (Figure 2b).

Overall, our results demonstrate that, after a 48-h incubation, CVTA, specifically CVTAP, performed best for detecting Pseudomonas in milk, whereas the common selective agars VRBA, MAC, and CPETRI were unable to detect all Pseudomonas isolates after 48 h. Our findings seem to conflict with those of GlueckChaloupka and White (2004), who found a stronger association $\left(\mathrm{R}^{2}=0.73\right)$ between VRBA without an overlay and poured SPC than observed here and weaker associations between SPC and CVTA at an incubation temperature of $32^{\circ} \mathrm{C}\left(\mathrm{R}^{2}=0.16\right.$; Glueck-Chaloupka and White, 2004). However, unlike our study, Glueck-Chaloupka and White (2004) evaluated the performance of each medium in detecting total psychrotolerant bacteria in milk, not Pseudomonas spp. specifically. Overall, our results indicate that CVTA is able to detect a diversity of strains and is therefore a suitable selective medium for the detection of post-pasteurization contamination by Pseudomonas spp. 

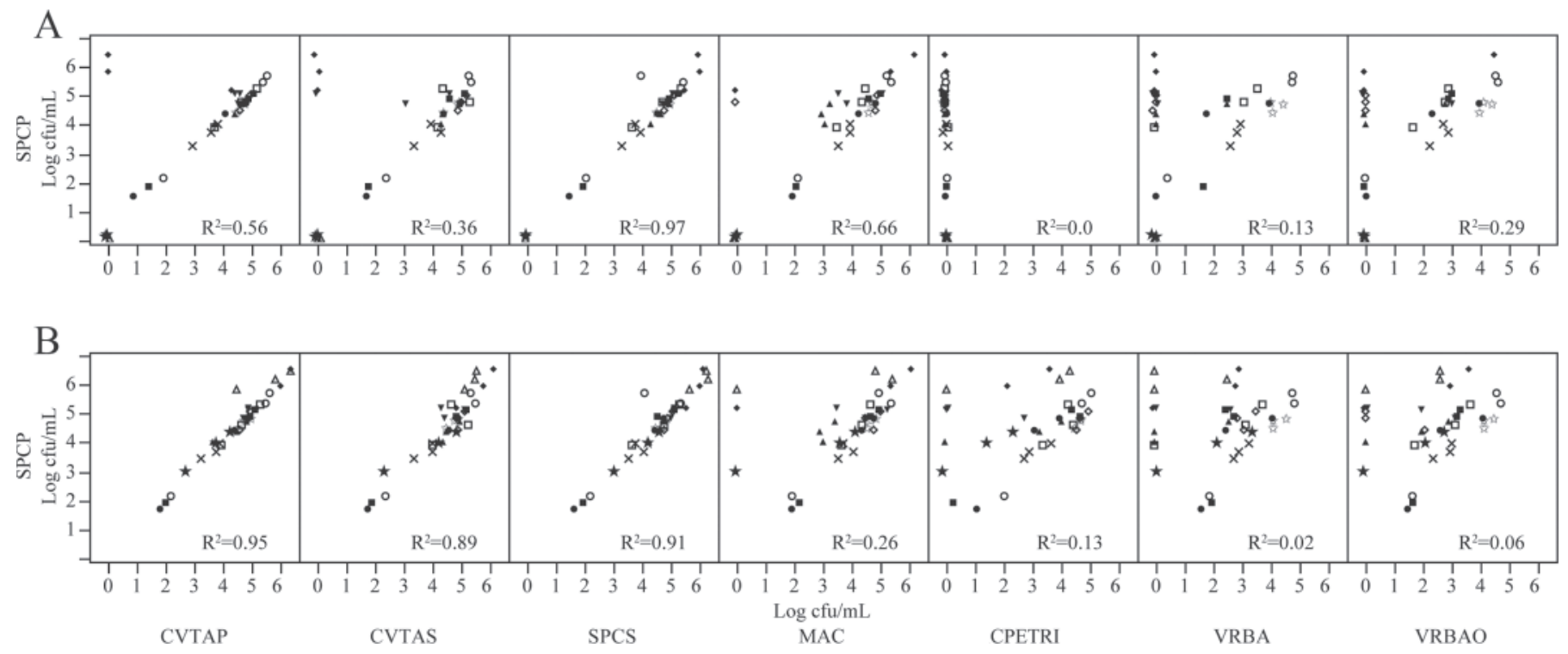

Figure 2. Scatter plot matrix of counts of Pseudomonas on standard plate count agar inoculated by pour plating (SPCP) versus crystal violet tetrazolium agar inoculated by pour plating (CVTAP), crystal violet tetrazolium agar inoculated by spiral plating (CVTAS), standard plate count agar inoculated by spiral plating (SPCS), MacConkey agar inoculated by spiral plating (MAC), 3M Coliform Petrifilm (CPETRI; 3M St. Paul, MN), violet red bile agar plates inoculated by pour plating (VRBA), and VRBA pour plates with an overlay (VRBAO) after $24 \mathrm{~h}$ (A) or $48 \mathrm{~h}$ (B) incubation at $32^{\circ} \mathrm{C}$. Symbols represent different Pseudomonas isolates: FSL R5-202 (open square), FSL R5-300 (filled square), FSL R5-318 (open circle), FSL R5-504 (filled circle), FSL R5-555 (open upward triangle), FSL R5-695 (filled upward triangle), FSL R5-779 (filled downward triangle), FSL W6-286 (open star), FSL W6-524 (filled star), FSL W6-987 (open diamond), FSL W7-098 (filled diamond), FSL W7-425 (sideways cross). All count data are presented in Supplementary Table S1 (available online at http://www.journalofdairyscience.org/).

\section{Reliable Detection of Pseudomonas spp. in Milk Requires a $48-h$ Incubation Time at $32^{\circ} \mathrm{C}$}

When counts were determined on different plating media after incubation for $24 \mathrm{~h}$ at $32^{\circ} \mathrm{C}$, detection efficiency on selective media was generally poor. Specifically, none of the selective media tested showed $\mathrm{R}^{2}$ values $>0.7$, and 4 out of 6 selective media showed $R^{2}$ $<0.4$ (Figure 2). These findings indicate that enumerating colonies after only a 24 -h incubation may result in inaccurate enumeration of Pseudomonas spp., which could allow post-pasteurization contamination with certain Pseudomonas spp. to go undetected.

After a 48-h incubation, improved detection efficiencies for certain selective media, particularly CVTA, were observed. Therefore, a 48-h incubation time resulted in an overall improvement in detection of Pseudomonas spp. in milk, which is consistent with the 48-h incubation times recommended for CPETRI and CVTA (Frank and Yousef, 2004) but not consistent with the recommended incubation time for MAC, which is 18 to $24 \mathrm{~h}$ (Difco, 1998). Our results suggest that plating milk samples on CVTA and incubating plates for $48 \mathrm{~h}$ is the best method for detecting Pseudomonas contamination in pasteurized milk. These findings support the method for the enumeration of gram-negative organisms in milk presented in Standard Methods for the Examination of Dairy Products (Frank and Yousef, 2004). However, whereas Standard Methods recommends incubating plates at $21^{\circ} \mathrm{C}$, our findings suggest that incubating plates at $32^{\circ} \mathrm{C}$ can accurately detect a diversity of Pseudomonas isolates in milk.

\section{Pseudomonas Isolates Differed in Their Abilities to Grow on Selective Media}

After $24 \mathrm{~h}$, we observed variability in growth among isolates on all media except CPETRI, which showed no detectable growth from any isolates. For example, FSL R5-555 and FSL W6-524 showed no detectable growth on any of the media after $24 \mathrm{~h}$, including SPCS and SPCP, and 2 additional isolates (i.e., FSL W6-987 and FSL W7-098) showed no growth on VRBA (Figure 2a).

Overall strain variability for growth on selective media was lower after $48 \mathrm{~h}$ than after $24 \mathrm{~h}$; for each selective media, no significant differences in the detected count was found in any of the 12 isolates tested (ANOVA; all $P$-values $>0.05)$, although some isolates showed variability among biological replicates. For example, after 48 h, FSL R5-555 and W6-524 each showed no growth for at least one biological replicate on MAC, CPETRI, VRBA, and VRBAO, although for each of these isolates, all 3 biological replicates produced growth on SPCP. This variability among replicates contributed to 
the low SPCP predictability for these selective media. These findings indicate that each of these isolates may require a longer incubation time than $48 \mathrm{~h}$ to reproducibly form colonies on MAC, CPETRI, VRBA, and VRBAO. In contrast, we observed less within-strain variability on CVTAP and CVTAS, as all isolates showed colony formation for all 3 replicates on both media.

The 12 isolates tested in our study varied in their abilities to grow (i.e., 48-h SPCP count minus inoculum count) in UHT milk at $6^{\circ} \mathrm{C}(P=0.0015$; ANOVA $)$. Not surprisingly, limited overall growth was observed in UHT milk at $6^{\circ} \mathrm{C}$ over $48 \mathrm{~h}$. Specifically, after $48 \mathrm{~h}, 7$ out of 12 isolates showed less than $1 \log$ growth, 4 out of 12 isolates showed between 1 and $2 \log$ growth, and W6-524, which is similar to Pseudomonas poae and the psychrotolerant species Pseudomonas lurida (Mishra et al., 2009) based on $16 \mathrm{~S}$ rDNA sequence similarity (Table 1) and phylogeny (Figure 1), showed the highest mean growth [2.63 (1.41 SD) log] after $48 \mathrm{~h}$ in UHT milk at $6^{\circ} \mathrm{C}$.

Overall, consistent with other results (De Jonghe et al., 2011), we demonstrated that different species of dairy-associated Pseudomonas vary in their abilities to grow in milk at refrigeration temperatures. We also demonstrated that Pseudomonas strains show variability in how quickly they form colonies on selective media. Furthermore, we have provided evidence that certain species of Pseudomonas are able to grow more rapidly than others in dairy products during refrigeration. These psychrotolerant Pseudomonas spp. may be present in natural environments (Mannisto and Haggblom, 2006; Selvakumar et al., 2009; Salwan et al., 2010), including soil (Mishra et al., 2009), which can be carried into processing plants through various routes. This highlights the importance of good in-plant sanitation practices in helping to prevent post-pasteurization contamination.

\section{CONCLUSIONS}

Our findings demonstrate that plating milk samples on CVTA (pour plates or spread plates) followed by a 48-h incubation at $32^{\circ} \mathrm{C}$ represents an effective method to recover a diversity of Pseudomonas spp. from refrigerated fluid milk. On the other hand, plating on 3M CPETRI and VRBA showed a limited ability to detect all common fluid-milk-associated Pseudomonas spp. Therefore, the use of coliform media as the lone indicator of gram-negative contamination, although convenient, is not an effective method for monitoring post-pasteurization contamination. Conversely, because general gram-negative selective media such as CVTA will not differentiate between coliforms and Pseudomonas spp., a program that includes testing for coliforms, using coliform selective media (e.g., VRBA or CPETRI), and general gram-negative spoilage organisms, using CVTA, is necessary to ensure the safety and quality of pasteurized dairy products, including fluid milk.

\section{ACKNOWLEDGMENTS}

The authors acknowledge the staff of the Milk Quality Improvement Program (MQIP) at Cornell University for their contributions to this work. This work was supported by the New York State Milk Promotion Advisory Board (through the New York State Department of Agriculture), representing New York State dairy farmers committed to producing high quality milk.

\section{REFERENCES}

Boran, R., and A. Ugur. 2010. Partial purification and characterization of the organic solvent-tolerant lipase produced by Pseudomonas fluorescens RB02-3 isolated from milk. Prep. Biochem. Biotechnol. 40:229-241.

Carey, N. R., S. C. Murphy, R. N. Zadoks, and K. J. Boor. 2005. Shelf lives of pasteurized fluid milk products in New York State: A tenyear study. Food Prot. Trends 25:102-113.

De Jonghe, V., A. Coorevits, K. Van Hoorde, W. Messens, A. Van Landschoot, P. De Vos, and M. Heyndrickx. 2011. Influence of storage conditions on the growth of Pseudomonas species in refrigerated raw milk. Appl. Environ. Microbiol. 77:460-470.

Difco. 1998. Difco Manual. 11th ed. Becton Dickinson and Company, Sparks, MD.

Durak, Z., H. Fromm, J. Huck, R. Zadoks, and K. Boor. 2006. Development of molecular typing methods for Bacillus spp. and Paenibacillus spp. isolated from fluid milk products. J. Food Sci. 71:M50-M56.

Eneroth, Å., S. Ahrnè, and G. Molin. 2000. Contamination routes of Gram-negative spoilage bacteria in the production of pasteurised milk, evaluated by randomly amplified polymorphic DNA (RAPD). Int. Dairy J. 10:325-331.

Frank, J. F., and A. E. Yousef. 2004. Tests for groups of microorganisms. Pages 227-248 in Standard Methods for the Examination of Dairy Products. H. M. Wehr and J. F. Frank, ed. American Public Health Association, Washington, DC.

Glueck-Chaloupka, A. A., and C. H. White. 2004. Comparison of three media used to estimate psychrotrophic bacterial counts in milk. Food Prot. Trends 24:77-81.

Huck, J. R., N. H. Woodcock, R. D. Ralyea, and K. J. Boor. 2007. Molecular subtyping and characterization of psychrotolerant endospore-forming bacteria in two New York State fluid milk processing systems. J. Food Prot. 70:2354-2364.

Jayarao, B. M., and L. Wang. 1999. A study on the prevalence of gramnegative bacteria in bulk tank milk. J. Dairy Sci. 82:2620-2624.

Mannisto, M. K., and M. M. Haggblom. 2006. Characterization of psychrotolerant heterotrophic bacteria from Finnish Lapland. Syst. Appl. Microbiol. 29:229-243.

Martin, N. H., S. C. Murphy, R. D. Ralyea, M. Wiedmann, and K. J. Boor. 2011. When cheese gets the blues: Pseudomonas fluorescens as the causative agent of cheese spoilage. J. Dairy Sci. 94:3176-3183.

Mishra, P. K., S. Mishra, S. C. Bisht, G. Selvakumar, S. Kundu, J. K. Bisht, and H. S. Gupta. 2009. Isolation, molecular characterization and growth-promotion activities of a cold tolerant bacterium Pseudomonas sp. NARs9 (MTCC9002) from the Indian Himalayas. Biol. Res. 42:305-313.

Nicodeme, M., J. P. Grill, G. Humbert, and J. L. Gaillard. 2005. Extracellular protease activity of different Pseudomonas strains: Dependence of proteolytic activity on culture conditions. J. Appl. Microbiol. 99:641-648. 
Ralyea, R. D., M. Wiedmann, and K. J. Boor. 1998. Bacterial tracking in a dairy production system using phenotypic and ribotyping methods. J. Food Prot. 61:1336-1340.

Ranieri, M. L., and K. J. Boor. 2009. Short communication: Bacterial ecology of high-temperature, short-time pasteurized milk processed in the United States. J. Dairy Sci. 92:4833-4840.

Salwan, R., A. Gulati, and R. C. Kasana. 2010. Phylogenetic diversity of alkaline protease-producing psychrotrophic bacteria from glacier and cold environments of Lahaul and Spiti, India. J. Basic Microbiol. 50:150-159.

Selvakumar, G., P. Joshi, P. K. Mishra, J. K. Bisht, and H. S. Gupta. 2009. Mountain aspect influences the genetic clustering of psychro- tolerant phosphate solubilizing Pseudomonads in the Uttarakhand Himalayas. Curr. Microbiol. 59:432-438.

Ternstrom, A., A. M. Lindberg, and G. Molin. 1993. Classification of the spoilage flora of raw and pasteurized bovine milk, with special reference to Pseudomonas and Bacillus. J. Appl. Bacteriol. $75: 25-34$.

Wiedmann, M., D. Weilmeier, S. S. Dineen, R. Ralyea, and K. J. Boor. 2000. Molecular and phenotypic characterization of Pseudomonas spp. isolated from milk. Appl. Environ. Microbiol. 66:2085-2095. 